\title{
Alternating sums of reciprocal generalized Fibonacci numbers
}

\author{
Kantaphon Kuhapatanakul
}

\begin{abstract}
Recently Holliday and Komatsu extended the results of Ohtsuka and Nakamura on reciprocal sums of Fibonacci numbers to reciprocal sums of generalized Fibonacci numbers. The aim of this work is to give similar results for the alternating sums of reciprocals of the generalized Fibonacci numbers with indices in arithmetic progression. Finally we note our generalizations of some results of Holliday and Komatsu.
\end{abstract}

Keywords: Fibonacci numbers; Reciprocal sums

AMS Subject Classification: Primary 11B37; secondary 11B39

\section{Introduction}

For a positive integer $p$, the generalized Fibonacci numbers $U_{n}$ are defined for $n \geq 0$ by

$$
U_{0}=0, U_{1}=1 \text { and } U_{n+2}=p U_{n+1}+U_{n} .
$$

If $p=1,2$, then $U_{n}$ are called the Fibonacci numbers $F_{n}$ and Pell numbers $P_{n}$, respectively. Also, if $p$ is any variable $x$, then $U_{n}$ are called Fibonacci polynomials $F_{n}(x)$.

(Ohtsuka and Nakamura 2008/2009) have found the formulas for the integer part of $\left(\sum_{k=n}^{\infty} \frac{1}{F_{k}}\right)^{-1}$ and $\left(\sum_{k=n}^{\infty} \frac{1}{F_{k}^{2}}\right)^{-1}$. (Holliday and Komatsu 2011) generalized these identities to the generalized Fibonacci numbers as follows

$$
\begin{aligned}
& \left\lfloor\left(\sum_{k=n}^{\infty} \frac{1}{U_{k}}\right)^{-1}\right\rfloor= \begin{cases}U_{n}-U_{n-1}, & \text { if } n \text { is even; } \\
U_{n}-U_{n-1}-1, & \text { if } n \text { is odd, }\end{cases} \\
& \left\lfloor\left(\sum_{k=n}^{\infty} \frac{1}{U_{k}^{2}}\right)^{-1}\right\rfloor= \begin{cases}p U_{n} U_{n-1}-1, & \text { if } n \text { is even; } \\
p U_{n} U_{n-1}, & \text { if } n \text { is odd, }\end{cases}
\end{aligned}
$$

where $\lfloor\cdot\rfloor$ is the floor function.
Similar properties were investigated in several different ways; see (Wu and Zhang 2012; Wu and Zhang 2013; Zhang 2011). Recently, (Kuhapatanakul 2013) gave a similar formula (1) for alternating sums of reciprocal generalized Fibonacci numbers.

In this paper we consider the alternating sums of reciprocals of the generalized Fibonacci numbers with indices in arithmetic progression and evaluate the integer part to the reciprocals of these sums. That is, we derive and prove the formulas of the following forms

$$
\left\lfloor\left(\sum_{k=n}^{\infty} \frac{(-1)^{k}}{U_{a k-b}}\right)^{-1}\right\rfloor \text { and }\left\lfloor\left(\sum_{k=n}^{\infty} \frac{(-1)^{k}}{U_{a k-b}^{2}}\right)^{-1}\right\rfloor,
$$

where $a, b$ are non-negative integers with $b<a$. We also extend two identities (1) and (2) by replacing $U_{k}$ with $U_{a k-b}$.

\section{Main results}

We begin with some identities of the generalized Fibonacci numbers whose will be used in the proofs of main theorem.

Lemma 1. Let $n, r$ be two integers with $n>r>0$. Then

(i) $U_{r} U_{n+1}+U_{r-1} U_{n}=U_{n+r}$.

(ii) $U_{r} U_{n-1}-U_{r-1} U_{n}=(-1)^{r-1} U_{n-r}$.

(iii) $U_{n}^{2}-U_{n-r} U_{n+r}=(-1)^{n-r} U_{r}^{2}$.

Proof. Every proof is done by induction and omitted.

\section{望 Springer}


Lemma 2. Let $n, r$ be two integers with $n>r>0$. Then

(i) $U_{n+r}-U_{n-r}>U_{r}^{2}$.

(ii) $U_{n+r}^{2}-U_{n-r}^{2}>\left|U_{n}^{4}-U_{n+r}^{2} U_{n-r}^{2}+U_{r}^{4}\right|$.

Proof. By using three identities of Lemma 1, we have

$$
\begin{aligned}
U_{n+r}-U_{n-r}= & U_{r} U_{n+1}+U_{r-1} U_{n} \\
& +(-1)^{r}\left(U_{r} U_{n-1}-U_{r-1} U_{n}\right) \\
= & U_{r}\left(U_{n+1}+(-1)^{r} U_{n-1}\right) \\
& +U_{r-1} U_{n}\left(1-(-1)^{r}\right) \\
> & U_{r}^{2}
\end{aligned}
$$

and

$$
\begin{aligned}
U_{n+r}^{2}-U_{n-r}^{2} & =\left(U_{n+r}-U_{n-r}\right)\left(U_{n+r}+U_{n-r}\right) \\
& =\left(U_{r} U_{n+1}+U_{r} U_{n-1}\right)\left(p U_{r} U_{n}+2 U_{r-1} U_{n}\right) \\
& >2 U_{r}^{2} U_{n}^{2} \\
& =\left|2(-1)^{n-r} U_{r}^{2} U_{n}^{2}-U_{r}^{4}+U_{r}^{4}\right| \\
& =\left|(-1)^{n-r} U_{r}^{2}\left(2 U_{n}^{2}-(-1)^{n-r} U_{r}^{2}\right)+U_{r}^{4}\right| \\
& =\left|\left(U_{n}^{2}-U_{n+r} U_{n-r}\right)\left(U_{n}^{2}+U_{n+r} U_{n-r}\right)+U_{r}^{4}\right| \\
& =\left|U_{n}^{4}-U_{n+r}^{2} U_{n-r}^{2}+U_{r}^{4}\right|,
\end{aligned}
$$

as desired.

We are now ready to verify our results.

Theorem 1. Let $a, b$ be two integers with $0 \leq b<a$ and $f(n)=a n-b$. Suppose $f(n-1)>0$ for all positive integer $n$. Then for $t=1,2$ we have

$$
\left\lfloor\left(\sum_{k=n}^{\infty} \frac{(-1)^{k}}{U_{f(k)}^{t}}\right)^{-1}\right\rfloor=\left\{\begin{array}{c}
(-1)^{n}\left(U_{f(n)}^{t}+U_{f(n-1)}^{t}\right) \\
\text { iff }(n-1)+n \text { is even; } \\
(-1)^{n}\left(U_{f(n)}^{t}+U_{f(n-1)}^{t}\right)-1 \\
\text { iff }(n-1)+n \text { is odd }
\end{array}\right.
$$

Proof. Since the proofs of both cases $t=1$ and $t=2$ are quite similar, we only give a proof for $t=2$. We see that $f(n)>a$ for all $n$. By Lemma 2(ii),

$$
U_{f(n+1)}^{2}-U_{f(n-1)}^{2}>\left|U_{f(n)}^{4}-U_{f(n+1)}^{2} U_{f(n-1)}^{2}+U_{a}^{4}\right|,
$$

we get

$$
\begin{aligned}
& \frac{(-1)^{n}}{U_{f(n)}^{2}+U_{f(n-1)}^{2}-(-1)^{n}}-\frac{(-1)^{n+1}}{U_{f(n+1)}^{2}+U_{f(n)}^{2}-(-1)^{n+1}} \\
& -\frac{(-1)^{n}}{U_{f(n)}^{2}}=\frac{(-1)^{n+1} U_{f(n-1)}^{2}+1}{U_{f(n)}^{2}\left(U_{f(n)}^{2}+U_{f(n-1)}^{2}-(-1)^{n}\right)} \\
& \quad+\frac{(-1)^{n}}{U_{f(n+1)}^{2}+U_{f(n)}^{2}+(-1)^{n}} \\
& >0
\end{aligned}
$$

Thus,

$$
\begin{aligned}
& \frac{1}{(-1)^{n}\left(U_{f(n)}^{2}+U_{f(n-1)}^{2}\right)-1} \\
& \quad>\frac{(-1)^{n}}{U_{f(n)}^{2}}+\frac{1}{(-1)^{n+1}\left(U_{f(n+1)}^{2}+U_{f(n)}^{2}\right)-1} .
\end{aligned}
$$

By applying the above inequality repeatedly, we obtain

$$
\sum_{k=n}^{\infty} \frac{(-1)^{k}}{U_{f(k)}^{2}}<\frac{1}{(-1)^{n}\left(U_{f(n)}^{2}+U_{f(n-1)}^{2}\right)-1}
$$

In a similar way, we have

$$
\begin{aligned}
& \frac{(-1)^{n}}{U_{f(n)}^{2}+U_{f(n-1)}^{2}+(-1)^{n}}-\frac{(-1)^{n+1}}{U_{f(n+1)}^{2}+U_{f(n)}^{2}+(-1)^{n+1}} \\
& -\frac{(-1)^{n}}{U_{f(n)}^{2}}=\frac{(-1)^{n+1} U_{f(n-1)}-1}{U_{f(n)}^{2}\left(U_{f(n)}^{2}+U_{f(n-1)}^{2}+(-1)^{n}\right)} \\
& +\frac{(-1)^{n}}{U_{f(n+1)}^{2}+U_{f(n)}^{2}-(-1)^{n}}
\end{aligned}
$$

$<0$

So

$$
\begin{aligned}
& \frac{1}{(-1)^{n}\left(U_{f(n)}^{2}+U_{f(n-1)}^{2}\right)+1} \\
& \quad<\frac{(-1)^{n}}{U_{f(n)}^{2}}+\frac{1}{(-1)^{n+1}\left(U_{f(n+1)}^{2}+U_{f(n)}^{2}\right)+1} .
\end{aligned}
$$

Repeating the above inequality, we obtain

$$
\frac{1}{(-1)^{n}\left(U_{f(n)}^{2}+U_{f(n-1)}^{2}\right)+1}<\sum_{k=n}^{\infty} \frac{(-1)^{k}}{U_{f(k)}^{2}} .
$$

Using Lemma 1(iii), we have

$$
\begin{gathered}
\frac{(-1)^{n}}{U_{f(n)}^{2}+U_{f(n-1)}^{2}}-\frac{(-1)^{n+1}}{U_{f(n+1)}^{2}+U_{f(n)}^{2}}-\frac{(-1)^{n}}{U_{f(n)}^{2}} \\
=\frac{(-1)^{n}\left(U_{f(n)}^{4}-U_{f(n-1)}^{2} U_{f(n+1)}^{2}\right)}{U_{f(n)}^{2}\left(U_{f(n)}^{2}+U_{f(n-1)}^{2}\right)\left(U_{f(n+1)}^{2}+U_{f(n)}^{2}\right)} \\
=\frac{(-1)^{f(n-1)+n} U_{a}^{2}\left(U_{f(n-1)} U_{f(n+1)}+U_{f(n)}^{2}\right)}{U_{f(n)}^{2}\left(U_{f(n)}^{2}+U_{f(n-1)}^{2}\right)\left(U_{f(n+1)}^{2}+U_{f(n)}^{2}\right)}
\end{gathered}
$$

The numerator of the right hand side of above equation is positive if $f(n-1)+n$ is even. Also, the numerator is negative if $f(n-1)+n$ is odd. 
Case I: If $f(n-1)+n$ is even, then

$$
\frac{(-1)^{n}}{U_{f(n)}^{2}+U_{f(n-1)}^{2}}>\frac{(-1)^{n}}{U_{f(n)}^{2}}+\frac{(-1)^{n+1}}{U_{f(n+1) f}^{2}+U_{f(n)}^{2}},
$$

so we obtain

$$
\sum_{k=n}^{\infty} \frac{(-1)^{k}}{U_{f(n)}^{2}}<\frac{(-1)^{n}}{U_{f(n)}^{2}+U_{f(n-1)}^{2}}
$$

Combining the (4) and (5), we get

$$
\begin{aligned}
\frac{1}{(-1)^{n}\left(U_{f(n)}^{2}+U_{f(n-1)}^{2}\right)+1} & <\sum_{k=n}^{\infty} \frac{(-1)^{k}}{U_{f(n)}^{2}} \\
& <\frac{1}{(-1)^{n}\left(U_{f(n)}^{2}+U_{f(n-1)}^{2}\right)},
\end{aligned}
$$

it is equivalent to

$$
\left\lfloor\left(\sum_{k=n}^{\infty} \frac{1}{U_{f(k)}^{2}}\right)^{-1}\right\rfloor=U_{f(n)}^{2}+U_{f(n-1)}^{2},
$$

where $f(n-1)+n$ is even.

Case II: If $f(n-1)+n$ is odd, then

$$
\frac{(-1)^{n}}{U_{f(n)}^{2}+U_{f(n-1)}^{2}}<\frac{(-1)^{n}}{U_{f(n)}^{2}}+\frac{(-1)^{n+1}}{U_{f(n+1)}^{2}+U_{f(n)}^{2}}
$$

We obtain

$$
\sum_{k=n}^{\infty} \frac{(-1)^{k}}{U_{f(k)}^{2}}>\frac{(-1)^{n}}{U_{f(n)}^{2}+U_{f(n-1)}^{2}}
$$

Combining the (3) and (6), we get

$$
\begin{aligned}
\frac{1}{(-1)^{n}\left(U_{f(n)}^{2}+U_{f(n-1)}^{2}\right)} & <\sum_{k=n}^{\infty} \frac{(-1)^{k}}{U_{f(k)}^{2}} \\
& <\frac{1}{(-1)^{n}\left(U_{f(n)}^{2}+U_{f(n-1)}^{2}\right)-1}
\end{aligned}
$$

it is equivalent to

$$
\left\lfloor\left(\sum_{k=n}^{\infty} \frac{(-1)^{k}}{U_{f(k)}^{2}}\right)^{-1}\right\rfloor=(-1)^{n}\left(U_{f(n)}^{2}+U_{f(n-1)}^{2}\right)-1,
$$

where $f(n-1)+n$ is odd.

This completes the proof.

\section{Remark 1.}

- If $f(n-1)+n=a(n-1)+n-b$ is even, then we have "both $a$ and $b$ are odd" or " $a, b, n$ are even" or " $a$ is even and $b, n$ are odd".
- If $(n-1)+n=a(n-1)+n-b$ is odd, then we have " $a$ is odd and $b$ is even" or " $a, b$ are even and $n$ is odd" or " $a, n$ are even and $b$ is odd".

Now we present some examples of Theorem 1 in the following corollary.

Corollary 1. For a positive integer $n>1$, we have

(i) $\left\lfloor\left(\sum_{k=n}^{\infty} \frac{(-1)^{k}}{U_{k}}\right)^{-1}\right\rfloor=(-1)^{n}\left(U_{n}+U_{n-1}\right)-1$.

(ii) $\left\lfloor\left(\sum_{k=n}^{\infty} \frac{(-1)^{k}}{U_{k}^{2}}\right)^{-1}\right\rfloor=(-1)^{n}\left(U_{n}^{2}+U_{n-1}^{2}\right)-1$.

(iii) $\left\lfloor\left(\sum_{\substack{k=n \\ \text { neven }}}^{\infty} \frac{(-1)^{k}}{U_{2 k}}\right)^{-1}\right\rfloor=U_{2 n}+U_{2 n-2}$.

(iv) $\left\lfloor\left(\sum_{\substack{k=n \\ \text { neven }}}^{\infty} \frac{(-1)^{k}}{U_{2 k-1}}\right)^{-1}\right\rfloor=U_{2 n-1}+U_{2 n-3}-1$.

Some examples for the Fibonacci numbers.

Example 1. For a positive integer $n>1$, we have

(i) $\left\lfloor\left(\sum_{k=n}^{\infty} \frac{(-1)^{k}}{F_{k}}\right)^{-1}\right\rfloor=(-1)^{n} F_{n+1}-1$.

(ii) $\left\lfloor\left(\sum_{k=n}^{\infty} \frac{(-1)^{k}}{F_{k}^{2}}\right)^{-1}\right\rfloor=(-1)^{n} F_{2 n-1}-1$.

\section{Sums of reciprocals}

We will obtain generalizations of the result of (Holliday and Komatsu 2011) on the reciprocal sums of $U_{k}$ to the reciprocal sums of $U_{a k-b}$.

Theorem 2. Let $a, b$ be two integers with $0 \leq b<a$ and $f(n)=a n-b$. Suppose $f(n-1) \geq 0$ for all positive integer $n$. Then for $t=1,2$ we have

$$
\left\lfloor\left(\sum_{k=n}^{\infty} \frac{1}{U_{f(k)}^{t}}\right)^{-1}\right\rfloor=\left\{\begin{array}{c}
U_{f(n)}^{t}-U_{f(n-1)}^{t} \\
\text { iff }(n-1) \text { is odd } \\
U_{f(n)}^{t}-U_{f(n-1)}^{t}-1 \\
\text { iff }(n-1) \text { is even. }
\end{array}\right.
$$

Proof. We only give a proof for $t=1$ as that of $t=2$ is similar. Since $f(n) \geq a$ for all $n$, we get

$$
U_{f(n+1)}-U_{f(n-1)}>U_{a}^{2}
$$


Using Lemma 1(i) and above inequality, we have

$$
\begin{aligned}
& \frac{1}{U_{f(n)}}-\frac{1}{U_{f(n)}-U_{f(n-1)}+1}+\frac{1}{U_{f(n+1)}-U_{f(n)}+1} \\
& =\frac{1-U_{f(n-1)} U_{f(n+1)}+U_{f(n)}^{2}+U_{f(n+1)}-U_{f(n-1)}}{U_{f(n)}\left(U_{f(n)}-U_{f(n-1)}+1\right)\left(U_{f(n+1)}-U_{f(n)}+1\right)} \\
& =\frac{(-1)^{f(n-1)} U_{a}^{2}+U_{f(n+1)}-U_{f(n-1)}+1}{U_{f(n)}\left(U_{f(n)}-U_{f(n-1)}+1\right)\left(U_{f(n+1)}-U_{f(n)}+1\right)} \\
& >0 .
\end{aligned}
$$

Thus, we obtain

$$
\sum_{k=n}^{\infty} \frac{1}{U_{f(k)}}>\frac{1}{U_{f(n)}-U_{f(n-1)}+1}
$$

Similarly, we can show that

$$
\sum_{k=n}^{\infty} \frac{1}{U_{f(k)}}<\frac{1}{U_{f(n)}-U_{f(n-1)}-1}
$$

On the other hand, we have

$$
\begin{aligned}
& \frac{1}{U_{f(n)}-U_{f(n-1)}}-\frac{1}{U_{f(n+1)}-U_{f(n)}}-\frac{1}{U_{f(n)}} \\
& =\frac{U_{f(n-1)} U_{f(n+1)}-U_{f(n)}^{2}}{U_{f(n)}\left(U_{f(n)}-U_{f(n-1)}\right)\left(U_{f(n+1)}-U_{f(n)}\right)} \\
& =\frac{(-1)^{f(n-1)-1} U_{a}^{2}}{U_{f(n)}\left(U_{f(n)}-U_{f(n-1)}\right)\left(U_{f(n+1)}-U_{f(n)}\right)} .
\end{aligned}
$$

The numerator of the right hand side of above equation is positive if $f(n-1)$ is odd. Also, the numerator is negative if $f(n-1)$ is even.

If $f(n-1)$ is odd, then we can verify that

$$
\sum_{k=n}^{\infty} \frac{1}{U_{f(k)}}<\frac{1}{U_{f(n)}-U_{f(n-1)}}
$$

and if $f(n-1)$ is even, then we get

$$
\sum_{k=n}^{\infty} \frac{1}{U_{f(k)}}>\frac{1}{U_{f(n)}-U_{f(n-1)}}
$$

Combining the (7) and (9), we obtain

$$
\frac{1}{U_{f(n)}-U_{f(n-1)}+1}<\sum_{k=n}^{\infty} \frac{1}{U_{f(k)}}<\frac{1}{U_{f(n)}-U_{f(n-1)}},
$$

it is equivalent to

$$
\left\lfloor\left(\sum_{k=n}^{\infty} \frac{1}{U_{a k-b}}\right)^{-1}\right\rfloor=U_{a n-b}-U_{a(n-1)-b},
$$

where $a(n-1)-b$ is odd.
Combining the (8) and (10), we obtain

$$
\frac{1}{U_{f(n)}-U_{f(n-1)}}<\sum_{k=n}^{\infty} \frac{1}{U_{f(k)}} \leq \frac{1}{U_{f(n)}-U_{f(n-1)}-1}
$$

it is equivalent to

$$
\left\lfloor\left(\sum_{k=n}^{\infty} \frac{1}{U_{a k-b}}\right)^{-1}\right\rfloor=U_{a n-b}-U_{a(n-1)-b}-1,
$$

where $a(n-1)-b$ is even.

\section{Remark 2}

- If $(n-1)=a(n-1)-b$ is odd, then we have " $a$ is even and $b$ is odd" or " $a, b, n$ are odd" or " $a$ is odd and $b, n$ are even".

- If $(n-1)=a(n-1)-b$ is even, then we have "both $a$ and $b$ are even" or " $a, b$ are odd and $n$ is even" or " $a, n$ are odd and $b$ is even".

Since $U_{n}^{2}-U_{n-1}^{2}=p U_{n} U_{n-1}-(-1)^{n}$, if we take $a=1$ in Theorem 2, we obtain the identities (1) and (2). Also, if we take $b=0$, then we get the same results given in ( $\mathrm{Wu}$ and Zhang 2013). In addition we have more results for $t=1,2$ such as

(i) $\left\lfloor\left(\sum_{k=n}^{\infty} \frac{1}{U_{2 k}^{t}}\right)^{-1}\right\rfloor=U_{2 n}^{t}-U_{2 n-2}^{t}-1$.

(ii) $\left\lfloor\left(\sum_{k=n}^{\infty} \frac{1}{U_{2 k-1}^{t}}\right)^{-1}\right\rfloor=U_{2 n}^{t}-U_{2 n-2}^{t}$.

(iii) $\left\lfloor\left(\sum_{k=n}^{\infty} \frac{1}{U_{3 k-1}^{t}}\right)^{-1}\right\rfloor=\left\{\begin{array}{c}U_{3 n-1}^{t}-U_{3 n-4}^{t}, \\ \text { if } n \text { is odd; } \\ U_{3 n-1}^{t}-U_{3 n-4}^{t}-1, \\ \text { if } n \text { is even. }\end{array}\right.$

(iv) $\left\lfloor\left(\sum_{k=n}^{\infty} \frac{1}{U_{3 k-2}^{t}}\right)^{-1}\right\rfloor=\left\{\begin{array}{c}U_{3 n-2}^{t}-U_{3 n-5}^{t}, \\ \text { if } n \text { is even; } \\ U_{3 n-2}^{t}-U_{3 n-5}^{t}-1, \\ \text { if } n \text { is odd. }\end{array}\right.$

Competing interests

The author declares that he has no competing interests.

\section{Acknowledgements}

This research is supported by a research grant for new scholars from the Thailand Research Fund (TRF), Grant No. MRG5680127, and the Kasetsart University Research and Development Institute (KURDI), Thailand. 
References

Holliday SH, Komatsu T (2011) On the sum of reciprocal generalized Fibonacci numbers. Integers 11A:A11

Kuhapatanakul K (2013) On the sums of reciprocal generalized Fibonacci numbers. J Integer Seq 16(Article 13.7.1):8

Ohtsuka H, Nakamura S (2008/2009) On the sum of reciprocal sums of Fibonacci numbers. Fibonacci Quart 46/47(2):153-159

Wu Z, Zhang W (2012) The sums of the reciprocals of the Fibonacci polynomials and Lucas polynomials. J Inequal Appl 134:8. doi:10.1186/1029-2424x-2012-134

Wu, Z, Zhang W (2013) Several identities involving the Fibonacci polynomials and Lucas polynomials. J Inequal Appl 205:14. doi:10.1186/1029-242X-2013-205

Zhang GJ (2011) The infinite sum of reciprocal of Fibonacci numbers. J Math Res Expo 31(6):1030-1034. doi:10.3770/j.issn:1000-344X.2011.06.010

\section{doi:10.1186/2193-1801-3-485}

Cite this article as: Kuhapatanakul: Alternating sums of reciprocal generalized Fibonacci numbers. SpringerPlus 2014 3:485.

Submit your manuscript to a SpringerOpen ${ }^{\circ}$ journal and benefit from:

- Convenient online submission

Rigorous peer review

- Immediate publication on acceptance

- Open access: articles freely available online

- High visibility within the field

- Retaining the copyright to your article

Submit your next manuscript at springeropen.com 